\title{
DIFFERENTIABLE MONOTONE MAPS ON MANIFOLDS. II $\left({ }^{1}\right)$
}

\author{
BY
}

\author{
P. T. CHURCH
}

\begin{abstract}
Let $M^{n}$ and $N^{n}$ be closed manifolds, and let $G$ be any (nonzero) module. (1) If $f: M^{3} \rightarrow N^{3}$ is $C^{3} G$-acyclic, then there is a closed $C^{3} 3$-manifold $K^{3}$ such that $N^{3} \# K^{3}$ is diffeomorphic to $M^{3}$, and $f^{-1}(y)$ is cellular for all but at most $r$ points $y \in N^{3}$, where $r$ is the number of nontrivial $G$-cohomology 3-spheres in the prime decomposition of $K^{3}$. (2) If $f: M^{3} \rightarrow M^{3}$ or $f: S^{3} \rightarrow M^{3}$ is $G$-acyclic, then $f$ is cellular. In case $G$ is $Z$ or $Z_{p}$ ( $p$ prime), results analogous to (1) and (2) in the topological category have been proved by Alden Wright. (3) If $f: M^{n} \rightarrow M^{n}$ or $f: S^{n} \rightarrow M^{n}$ is real analytic monotone onto, then $f$ is a homeomorphism.
\end{abstract}

1. Conventions and definitions. Let $G$ be a module, and let $A \subset M^{n}$ be compact. Then $A$ is acyclic ( $k$-acyclic; cellular) is the reduced Čech cohomology module $\tilde{H}^{*}(A ; G)=0\left(H^{k}(A ; G)=0\right.$; there are $n$-cells $A_{k} \subset M^{n}$ such that $A_{k+1} \subset$ int $A_{k}$ and $\bigcap_{k} A_{k}=A$ ). A proper onto map $f: M^{n} \rightarrow N^{n}$ is monotone (acyclic; cellular) if, for each $y \in N^{n}, f^{-1}(y)$ is connected (acyclic; cellular). The branch set $B_{f}$ is the set of points in $M^{n}$ at which $f$ fails to be a local homeomorphism.

Standing hypotheses. Unless otherwise specified, all manifolds are connected, separable, and without boundary. Whenever the statement of a theorem refers to a map $f$ without specifying its domain and range, it is understood that $f: M^{n} \rightarrow N^{n}$ is proper and onto. Whenever coefficients of cohomology are not specified, any (nonzero) module $G$ may be used.

Other conventions and definitions are as in [6, pp. 185-186]. For other work on monotone, acyclic, and cellular maps $f: M^{n} \rightarrow N^{n}$, see bibliographies of this paper, [1], [15], and (vast) [30].

2. Real analytic monotone maps.

2.1. Definition. Let $G$ be a module, and let

$$
C_{f}=C_{f}(G)=f^{-1}\left(\mathrm{Cl}\left\{y \in N^{n}: \tilde{H}^{*}\left(f^{-1}(y) ; G\right) \neq 0\right\}\right) .
$$

2.2. LEMMA. Let $L$ be a principal ideal domain, let $M^{n}$ be orientable over $L$, and let $\operatorname{dim}\left(f\left(C_{f}\right)\right) \leqq 0$.

Received by the editors August 26, 1970.

AMS 1970 subject classifications. Primary 57A60, 54C10; Secondary 57D35, 32C05.

Key words and phrases. Monotone, acyclic, cellular, open, differentiable, real analytic maps.

( $\left.{ }^{1}\right)$ Research supported in part by an NSF Senior Postdoctoral Fellowship at the Institute for Advanced Study during 1965-66. 
(1) If $M^{n}$ and $N^{n}$ are closed, then there is a $k(k=0,1, \ldots)$ such that $f\left(C_{f}\right)$ has exactly $k$ points and

$$
k \leqq \sum_{r>0}\left(\text { number of generators of } H^{r}\left(M^{n}\right)\right)
$$

in fact, if $L$ is a field,

$$
k \leqq \sum_{r>0}\left(\operatorname{dim}\left(H^{r}\left(M^{n}\right)\right)-\operatorname{dim}\left(H^{r}\left(N^{n}\right)\right)\right)
$$

(2) If $B^{n} \subset N^{n}$ is a closed $n$-cell with $\partial B^{n} \cap f\left(C_{f}\right)=\varnothing$, then inclusion induces an isomorphism

$$
H^{r}\left(f^{-1}\left(B^{n}\right)\right) \approx \sum\left\{H^{r}\left(f^{-1}(y)\right): y \in B^{n}\right\}
$$

for every $r>0$.

Proof. From [13, p. 639, Theorem 3, (2)]

$$
0 \rightarrow H_{c}^{r}\left(N^{n}\right) \rightarrow H_{c}^{r}\left(M^{n}\right) \rightarrow \sum\left\{H^{r}\left(f^{-1}(y)\right): y \in N^{n}\right\} \rightarrow 0
$$

is split exact for $r>0$, and (1) follows. Note that the numbers of generators of $H^{r}\left(M^{n}\right)$ and $H^{r}\left(N^{n}\right)$ are finite [27, p. 342, (11)].

From the exactness of

$$
0 \rightarrow H_{c}^{r}\left(\text { int } B^{n}\right) \rightarrow H_{c}^{r}\left(f^{-1}\left(\text { int } B^{n}\right)\right) \rightarrow \sum\left\{H^{r}\left(f^{-1}(y)\right): y \in \text { int } B^{n}\right\} \rightarrow 0
$$

for $r>0$ it follows that $H_{c}^{r}\left(f^{-1}\left(\right.\right.$ int $\left.\left.B^{n}\right)\right) \approx \sum H^{r}\left(f^{-1}(y)\right)$ for $1 \leqq r \leqq n-1$ and $\sum H^{n}\left(f^{-1}(y)\right)=0$. From the Vietoris Mapping Theorem [27, p. 346, (18)] $H^{r}\left(f^{-1}\left(\partial B^{n}\right)\right) \approx H^{r}\left(\partial B^{n}\right)$, and from the cohomology sequence

$$
\cdots \leftarrow H^{r}\left(f^{-1}\left(\partial B^{n}\right)\right) \leftarrow H^{r}\left(f^{-1}\left(B^{n}\right)\right) \leftarrow H_{c}^{r}\left(f^{-1}\left(\text { int } B^{n}\right)\right) \leftarrow H^{r-1}\left(f^{-1}\left(\partial B^{n}\right)\right) \leftarrow \cdots
$$

(2) follows.

2.3. Lemma. Let $L$ be a principal ideal domain, let $M^{n}$ be orientable over $L$, and let $f$ be $C^{n}$ monotone but not acyclic. Then $\operatorname{dim} C_{f}>\operatorname{dim} f\left(C_{f}\right)$.

Proof. Suppose $\operatorname{dim} f\left(C_{f}\right)=0$. If $\operatorname{dim} C_{f} \leqq 0$, then $f$ is acyclic; thus $\operatorname{dim} C_{f}>0$.

Thus we may suppose that $\operatorname{dim} f\left(C_{f}\right)=q>0$. By [11, (3.7), (3.5), and (3.8)] there are a $C^{\infty}$ closed manifold $J^{m}$, a $C^{\infty}$ map $h: J^{m} \times R^{n-m} \rightarrow S^{m} \times R^{n-m}$ with $h\left(J^{m} \times\{t\}\right) \subset S^{m} \times\{t\} \quad(m=0,1, \ldots, n-q$; thus $n-m \geqq q \geqq 1)$, a $C^{\infty}$ embedding $\gamma$ of $J^{m} \times R^{n-m}$ in $M^{n}$, and a $C^{\infty}$ embedding $\delta$ of $S^{m} \times R^{n-m}$ in $N^{n}$ such that

(a) $\delta^{-1} h \gamma$ is the restriction of $f$,

(b) $f\left(C_{f}\right) \cap$ imag $\delta \neq \varnothing$, and

(c) $\operatorname{dim}\left(f\left(B_{f}\right) \cap \delta\left(S^{m} \times\{t\}\right)\right) \leqq 0$ for every $t \in R^{n-m}$.

Let $h_{t}: J^{m} \times\{t\} \rightarrow S^{m} \times\{t\}$ be the restriction of $h$; clearly $\bigcup_{t} C\left(h_{t}\right) \subset C_{h}$, so that (from (c)) each $\operatorname{dim} h_{t}\left(C\left(h_{t}\right)\right) \leqq 0$ and 2.2 (1) applies to $h_{t}$ yielding an integer $k(t) \geqq 0$. There is a $t$ such that $k(t)$ is maximal for $t \in R^{n-m}$, and we may suppose 
that $t=0$. Let $y_{j}(j=1,2, \ldots, s)(s=k(0))$ be exactly those points in $S^{m}$ such that $H^{r}\left(h^{-1}\left(y_{j}, 0\right)\right) \neq 0$ for some $r>0$.

The proof now follows that given in [6, pp. 201-202], beginning with the third paragraph, with the following changes: $h, m, L, C_{h}$, and $C_{e}$ in place of $f, p, Z, B_{f}$, and $B_{e}$, respectively; $S^{m}$ in place of $B^{p}$, int $B^{p}$, and $\Sigma$; references to (1) should be omitted, and (2) should be interpreted as 2.2 (2). At the end we observe that $\operatorname{dim} C_{f} \geqq \operatorname{dim} C_{h} \geqq n-m+i>n-m \geqq q=\operatorname{dim} f\left(C_{f}\right)$.

2.4. Corollary. Let $f$ be real analytic monotone, but not a homeomorphism. Then $\operatorname{dim} B_{f}>\operatorname{dim} f\left(B_{f}\right)$.

Proof. Use $Z_{2}$ coefficients. The map $g: M^{n}-C_{f} \rightarrow N^{n}-f\left(C_{f}\right)$ defined by restriction of $f$ is acyclic, and thus a homeomorphism $([10$, p. $33,(6.1)]$; the conclusion should be "is a homeomorphism"). Then $C_{f}=B_{f}$, and the conclusion results from (2.3).

2.5. LEMMA. Let $f$ be real analytic monotone, but not necessarily proper. Then $B_{f}$ is a $C$-analytic subset of $M^{n}$.

The proof follows from the first five paragraphs of the proof of $[10$, p. 31, (5.4)]; in particular, from the first sentence of the sixth paragraph.

2.6. LemMA. Let $M^{n}$ and $N^{n}$ be closed, and let $f$ be real analytic monotone with $\operatorname{dim} B_{f}=q \geqq 0$. Then

$$
\operatorname{dim}\left(H^{q}\left(N^{n} ; Z_{2}\right)\right)<\operatorname{dim}\left(H^{q}\left(M^{n} ; Z_{2}\right)\right) .
$$

Proof. Suppose the contrary. By $2.4 q>0$, by 2.5 and [3] $H^{q}\left(B_{f}\right) \neq 0$, and by $2.4 H^{q}\left(f\left(B_{f}\right)\right)=0$. Since $f$ maps $M^{n}-B_{f}$ homeomorphically onto $N^{n}-f\left(B_{f}\right)$, it induces [27, p. 318, (5)] $H^{k}\left(N^{n}, f\left(B_{f}\right)\right) \approx H^{k}\left(M^{n}, B_{f}\right)$ and [6, p. 192, (2.13)] a monomorphism of $H^{k}\left(N^{n}\right)$ into $H^{k}\left(M^{n}\right)(k=0,1, \ldots)$. Since $H^{k}\left(M^{n}\right)$ and $H^{k}\left(N^{n}\right)$ are finite dimensional $[27$, p. $342,(11)]$, it follows from the contrary assumption that the monomorphism is an isomorphism (onto) if $k=q$; a contradiction results from the Five Lemma [14, p. 14, (3.3)(ii)] applied to the cohomology sequences of $\left(M^{n}, B_{f}\right)$ and $\left(N^{n}, f\left(B_{f}\right)\right)$.

2.7. COROLlary. If $M^{n}=S^{n}$ and $f$ is real analytic monotone, then $f$ is a homeomorphism.

Proof. Since $f$ is continuous, $N^{n}$ is compact, i.e. closed.

2.8. CoROllaRY. If $M^{n}$ is closed, a monotone real analytic map $f: M^{n} \rightarrow M^{n}$ is a homeomorphism.

2.9. CoRollary. If $M^{n}$ and $N^{n}$ are closed, and $f: M^{n} \rightarrow N^{n}$ and $g: N^{n} \rightarrow M^{n}$ are real analytic and monotone, then $f$ and $g$ are homeomorphisms.

Statements corresponding to $2.7,2.8$, and 2.9 for $C^{\infty}$ maps are false, in fact $f$ need not be acyclic [6, p. 192, (2.12)]. Results somewhat analogous for $n=3$ to 2.8 and 2.9 are given in [18], and to 2.7 in [2]. 
2.10. Remark. The author does not know whether, for $M^{n}$ and $N^{n}$ closed, a real analytic monotone (onto) map $f: M^{n} \rightarrow N^{n}$ is necessarily a homeomorphism (see $[10, \S 6]$ ). Suppose that there is such a counterexample, and that $n$ is the smallest dimension for which it occurs. Then $n \geqq 2$, and $f^{-1}(y)$ is a single point except for $s$ points $y_{i} \in N$, where

$$
s \leqq \operatorname{dim}\left(H^{*}\left(M^{n} ; Z_{2}\right)\right)-\operatorname{dim}\left(H^{*}\left(N^{n} ; Z_{2}\right)\right) .
$$

In fact, $M^{n}$ is homeomorphic to the connected sum of $N^{n}$ and $K_{i}(i=1,2, \ldots, r)$ where no $K_{i}$ is homeomorphic to $S^{n}$.

Proof. If $n=1$, then $M^{1}=S^{1}$. If for some $y \in N^{1}$, the $C$-analytic set $f^{-1}(y)$ is not a single point, then $f^{-1}(y)=S^{1}$, contradicting the ontoness of $f$. Thus $n \geqq 2$.

Let $y_{1}$ and $y_{2}$ be two distinct points of $f\left(B_{f}\right)$, let $\alpha: R^{n} \rightarrow N^{n}$ be a real analytic embedding with $\alpha(0)=y_{1}$ and $y_{2} \notin$ imag $\alpha$, and let $g: f^{-1}\left(\alpha\left(R^{n}\right)\right) \rightarrow R^{n}$ be the restriction of $\alpha^{-1} \circ f$. There is a $C^{n}$ embedding $\mu: S^{n-1} \rightarrow R^{n}$ such that $d(\mu$, id $)<\frac{1}{2}$ in the (coarse-fine) $C^{n}$ topology [20, p. 25] and $g$ is transverse regular on $\mu\left(S^{n-1}\right)$ ([29, p. 26] and [8, p. 376, (2.6)]), and there is a $\delta, 0<\delta<\frac{1}{2}$, such that if $\nu: S^{n-1} \rightarrow R^{n}$ is a $C^{n}$ embedding with $d(\mu, v)<\delta$, then $f$ is transverse regular on $v\left(S^{n-1}\right)([29, \mathrm{p}$. 27] and [8, p. 376, (2.6)]). By [31, p. 654, Theorem 2] there is a real analytic embedding $\nu: S^{n-1} \rightarrow R^{n}$ such that $d(\mu, \nu)<\delta$. For $r>0$ let $\nu_{r}$ be the embedding defined by $\nu_{r}(x)=r \cdot \nu(x)$; for $r$ in some open interval $J$ about $1, d\left(\mu, \nu_{r}\right)<\delta$ also. Thus $g^{-1}\left(\nu_{r}\left(S^{n-1}\right)\right)=L^{n-1}$ is a real analytic $(n-1)$-manifold also [29, p. 23], and by the minimality of $n, g \mid L^{n-1}: L^{n-1} \rightarrow \nu_{r}\left(S^{n-1}\right)$ is a homeomorphism for all $r \in J$. The open set $V=\bigcup\left\{v_{r}\left(S^{n-1}\right): r \in J\right\}$ has compact closure and separates $R^{n}$ with 0 in a bounded component of $R^{n}-V$. It follows that $y_{1}$ and $y_{2}$ are in different components of $f\left(B_{f}\right)$. Since $y_{1}$ and $y_{2}$ were arbitrary, $f\left(B_{f}\right)$ is a closed 0-dimensional set.

By [3], if $f^{-1}(y)$ is $Z_{2}$-acyclic, it is a single point; the second conclusion follows from 2.2(1).

For the third conclusion, there are (as above) mutually disjoint real analytic $n$-balls $B_{i} \subset N^{n}$ such that $y_{i} \in$ int $B_{i}$ and $f$ is transverse regular on $\partial B_{i}$; thus $f^{-1}\left(\partial B_{i}\right)$ is homeomorphic to $S^{n-1}$, and the conclusion results from 2.2(1).

3. Acyclic maps. In this section the cohomology coefficient module $(G \neq 0)$ is arbitrary, unless otherwise stated.

3.1. Lemma. Let $K^{m}$ be a closed m-manifold $(m=2$ or 3$)$, and let $h: K^{m} \rightarrow S^{m}$ be acyclic. Then $K^{m}$ is orientable, and $K^{2}$ is homeomorphic to $S^{2}$.

Proof. By the Vietoris Mapping Theorem [27, p. 346, (18)]

$$
h^{*}: \tilde{H}^{j}\left(S^{m} ; G\right) \approx \tilde{H}^{j}\left(K^{m} ; G\right),
$$

and $K$ is a finite polyhedron.

Suppose that $K^{m}$ is nonorientable. Then $H^{m}\left(K^{m} ; Z\right) \approx Z_{2}$, and from the naturality 
of the Universal Coefficient Theorem [27, p. 236, (1)], some homomorphism

$$
\alpha \otimes 1: Z \otimes G \rightarrow Z_{2} \otimes G
$$

is an isomorphism; thus, for every $g \in G, 2 g=0$. From [27, p. 206, E.2] $H_{m-1}\left(K^{m} ; Z\right)$ has a summand of $Z_{2}$, and hence Hom $\left(H_{m-1}\left(K^{m} ; Z\right), G\right) \neq 0$. Since $H^{m-1}\left(K^{m} ; G\right)$ $=0$, a contradiction results $[27$, p. $243,(3)]$.

Since $H^{1}\left(K^{2} ; G\right)=0$, the second conclusion is immediate from the classification of closed orientable 2-manifolds [27, p. 148-149].

3.2. REMARK. For any closed 3-manifold $M^{3}$ there is a smallest natural number $p\left(M^{3}\right)$ such that, if $M^{3}$ is homeomorphic to the connected sum of closed 3manifolds $K_{i}\left(i=1,2, \ldots, p\left(M^{3}\right)+1\right)$, then at least one of the $K_{i}$ is homeomorphic to $S^{3}$ [12, p. 253]. In case $M^{3}$ is orientable, $M^{3}$ actually has a unique decomposition into primes [19].

3.3. Proposition. Let $f$ be a $C^{3}$ proper monotone map, and let $Y \subset N^{n}$ be the smallest closed subset such that $f^{-1}(y)$ is cellular for each $y \in N^{n}-Y$.

(a) Then $\operatorname{dim} Y \leqq n-2$.

(b) If $H^{1}\left(f^{-1}(y)\right)=0$ except for a discrete set of $y \in N^{n}$, then

(i) $\operatorname{dim} Y \leqq n-3$, and

(ii) for every open set $U \subset N^{n}$ with $\pi_{1}(U)=0, \pi_{1}(U-Y)=0$.

(c) If, in addition, $n=3$, then $Y$ is discrete.

(d) If, in addition, $M^{3}$ is closed, then there is a $C^{3}$ closed 3-manifold $K^{3}$ such that $M^{3}$ is diffeomorphic to $N^{3} \# K^{3}$ and the number of points $\nu(Y) \leqq p\left(K^{3}\right) \leqq p\left(M^{3}\right)$.

(If $M^{n}$ is orientable, then $p\left(K^{3}\right)=p\left(M^{3}\right)-p\left(N^{3}\right)$ (Remark 3.2).)

Proof. Conclusion (a) results from [6, p. 189, (2.6)] and [9, Proposition 4].

Suppose that (b) is false. Then [11, p. 80, (3.7)] there is a $C^{3}$ embedding $\lambda: S^{m} \times R^{n-m} \rightarrow N^{n}(m=0,1$, or 2$)$ such that $f$ is transverse regular on $\lambda\left(S^{m} \times\{t\}\right)$ and $\lambda\left(S^{m} \times\{t\}\right) \cap Y \neq \varnothing$ for each $t \in R^{n-m}$. By [11, p. 80, (3.5)] there are $\varepsilon>0$, a $C^{3}$ closed $m$-manifold $L^{m}$, and a diffeomorphism $\sigma$ of $L^{m} \times S(0, \varepsilon)$ onto $f^{-1}\left(\lambda\left(S^{m} \times S(0, \varepsilon)\right)\right)$ such that $\lambda^{-1} f \sigma\left(L^{m} \times\{t\}\right) \subset S^{m} \times\{t\}$ for each $t \in S(0, \varepsilon)$. From [6, (2.6)], $m=2$. Let $g=\lambda^{-1} f_{\sigma}$.

Since $f$ is monotone onto, $L^{2}$ is connected [32, p. 138, (2.2)] and $g\left(L^{2} \times\{t\}\right)$ $=S^{2} \times\{t\}$; thus for every $(w, t) \in S^{2} \times S(0, \varepsilon), g^{-1}(w, t) \neq L^{2} \times\{t\}$, so that $H^{2}\left(g^{-1}(w, t)\right)=0$. Since $H^{1}\left(f^{-1}(y)\right)=0$ except for a discrete set of $y \in N^{n}$, we may suppose that $H^{1}\left(g^{-1}(w, t)\right)=0$ for every $(w, t) \in S^{2} \times S(0, \varepsilon)$; thus $g$ is acyclic.

By $3.1 L^{2}$ is homeomorphic to $S^{2}$. From $\left[6\right.$, p. 191, (2.11)] each $H^{1}\left(g^{-1}(w, t) ; Z_{2}\right)$ $=0$, so that $H_{1}\left(g^{-1}(w, t) ; Z_{2}\right)=0$. For each open subset $U$ of $L^{2} \times S(0, \varepsilon)$ with $g^{-1}(w, t) \subset U$, there is [24, p. 853, Lemma 2] a closed 2-cell $D \subset U \cap\left(K^{2} \times\{t\}\right)$ with $g^{-1}(w, t) \subset$ int $D$. Since there is a $\delta>0$ with $D \times S(t, \delta) \subset U, g^{-1}(w, t)$ is cellular in $L^{2} \times S(0, \varepsilon)$, and a contradiction results. Thus (b) is proved.

Now suppose, in addition, that $n=3$. Let $\bar{y} \in Y$ and let $B^{3}$ be a $C^{3} 3$-ball with $\bar{y} \in$ int $\left(B^{3}\right)$; we may suppose that $H^{1}\left(f^{-1}(y)\right)=0$ for each $y \in \partial B^{3}$ and that $f$ is 
transverse regular on $\partial B^{3}\left([29\right.$, p. 26$]$ and $\left[8\right.$, p. 376, (2.6)]). By $\left[29\right.$, p. 23] $f^{-1}\left(\partial B^{3}\right)$ is a $C^{3} 2$-manifold, and it follows as above that $f^{-1}(\partial B)$ is homeomorphic, and thus $C^{3}$ diffeomorphic, to $S^{2}$.

Let $L^{3}\left(T^{3}\right)$ be the $C^{3}$ 3-manifold obtained from $f^{-1}\left(B^{3}\right)\left(B^{3}\right)$ by identifying $f^{-1}\left(\partial B^{3}\right)(\partial B)$ to a point $a(b)$. Let $g: L^{3} \rightarrow T^{3}$ be the map defined by $g(a)=b$ and $g \mid f^{-1}$ (int $\left.B^{3}\right)=f \mid f^{-1}$ (int $B^{3}$ ); since $g$ is $C^{3}$ except on $g^{-1}(b)=\{a\}$, we may suppose $[6$, p. $189,(2.4)]$ that $g$ is $C^{3}$. Thus to prove that $\bar{y}$ is an isolated point of $Y$, and hence prove (c), it suffices to prove (d) for $g$.

Thus we may suppose that $M^{3}$ is closed; hence $N^{3}$ is closed. Let $y_{i}(i=1,2, \ldots, s)$ be distinct points of $Y$; choose as above $C^{3}$ 3-balls $B_{i}^{3}$ with $y_{i} \in$ int $\left(B_{i}^{3}\right), f$ transverse regular on $\partial B_{i}^{3}$, and the $B_{i}^{3}$ mutually disjoint. From the definition of $Y$ we may suppose that no $f^{-1}\left(B_{i}^{3}\right)$ is a 3-cell. Let $K_{i}^{3}$ be the $C^{3}$ manifold obtained by attaching a copy of the unit 3-ball $D^{3}$ to $f^{-1}\left(B_{i}^{3}\right)$ by a diffeomorphism of their boundaries; let $P^{3}$ be similarly obtained from $M^{3}-\bigcup_{i} f^{-1}$ (int $B_{i}^{3}$ ) by attaching $s$ copies of $D^{3}$. Let $K_{i}^{3}$ be the connected sum of the $K_{i}^{3}$; then $M^{3}$ is diffeomorphic to $P^{3} \# K^{3}$. It follows from 3.2 that $s \leqq p\left(K^{3}\right)$, which is $p\left(M^{3}\right)-p\left(N^{3}\right)$ if $M^{3}$ (and hence $N^{3}$ ) is orientable.

Hence we may suppose that $y_{i}(i=1,2, \ldots, s)$ are all the points of $Y$. Define $h: P^{3} \rightarrow N^{3}$ by $h(x)=f(x)$ for $x \in M^{3}-\bigcup_{i} f^{-1}$ (int $\left.B_{i}^{3}\right)$, and on the balls it is the cone map. Then $h$ is cellular, $P^{3}$ is homeomorphic to $N^{3}$ [1], and thus [21] diffeomorphic to $N^{3}$ (or simply use [6, p. 189, (2.4)] and 3.8).

3.4. ExAmples. Let $f: M^{3} \rightarrow S^{3}$ be the example of $[6,(4.5)]$, and let 1 be the identity map on $S^{n-3}$; then $\operatorname{dim} Y=n-3$ for $f \times 1$. In (b) some hypothesis is needed [6, (2.12)].

3.5. Corollary. Let $M^{3}$ and $N^{3}$ be closed, and let $f$ be a $C^{3}$ acyclic map. Then there is a cohomology 3-sphere $K^{3}$ such that $N^{3} \# K^{3}$ is diffeomorphic to $M^{3}$ and $f^{-1}(y)$ is cellular for all but at most $p\left(K^{3}\right)$ points $y \in N^{3}$, where $p\left(K^{3}\right)$ is the number of nontrivial cohomology 3-spheres in the prime decomposition of $K^{3}$.

Proof. In the above proof it is immediate from 3.1 and the Vietoris Mapping Theorem applied to $g$ that $K_{i}^{3}$ are orientable and cohomology 3-spheres. Thus $K^{3}$ and every summand of $K^{3}$ are (orientable) cohomology 3-spheres.

3.6. Corollary. Let $M^{3}$ and $N^{3}$ be closed, and let $f: M^{3} \rightarrow N^{3}$ and $g: \dot{N}^{3} \rightarrow M^{3}$ be $C^{3}$ acyclic. Then $f$ and $g$ are cellular, and $M^{3}$ and $N^{3}$ are diffeomorphic.

Proof. By $3.5 M^{3} \approx N^{3} \# K^{3}$ and $N^{3} \approx M^{3} \# L^{3}$, so that $M^{3} \approx M^{3} \# K^{3} \# L^{3}$. From $3.2 K^{3} \approx S^{3} \approx L^{3}$, and the conclusion results from 3.5 .

3.7. Corollary. Let $M^{3}$ be closed, and let $f: M^{3} \rightarrow M^{3}$ be $C^{3}$ acyclic. Then $f$ is cellular.

If $M^{3}$ is orientable in 3.7 and 3.8, then 1-acyclic suffices.

3.8. Corollary. If $f: S^{3} \rightarrow M^{3}$ is $C^{3} 1$-acyclic, then $f$ is cellular and $M^{3} \approx S^{3}$. 
Alden Wright [33], [34] has proved analogs of 3.3(c), (d), 3.5, 3.6, 3.7, and 3.8: he assumes no differentiability hypothesis, but instead of 1-acyclic over an arbitrary coefficient module, he assumes strongly 1 -acyclic over $Z$ or $Z_{p}$ ( $p$ prime). In addition the techniques in the proof (of 3.3) are quite different from his.

Corollaries 3.5, 3.6, and 3.7 are somewhat analogous to results of McMillan [18], and 3.8 is analogous to [17, Theorems 2 and 3] (note that the coefficient module is arbitrary in our case). Actually the author proved $3.2,[11$, p. 80, (3.7)] and 3.5 in the spring of 1966 , but he has been tardy about publishing them. See also McMillan [16, pp. 134-135, Theorem 5 and Addendum 2], and [15, Corollary 3.5] (see [17]) and Armentrout [1] and [2].

Armentrout [1] has proved (essentially) the following result in the topological category, where it is quite deep. (See also [26].) We now observe that it can be easily obtained in the differential category.

3.9. Proposition. Let $f: M^{3} \rightarrow N^{3}$ be $C^{3}$ cellular, and let $\varepsilon: N^{3} \rightarrow(0, \infty)$ be continuous. Then there is a $C^{3}$ diffeomorphism $h: M^{3} \rightarrow N^{3}$ such that $d(f(x), h(x))$ $<\varepsilon(f(x))$ for each $x \in M^{3}$.

Proof. We may suppose that $\varepsilon$ is sufficiently small that, for each $y \in N^{3}$, $f^{-1}(S(y, \varepsilon(y)))$ is contained in a closed 3-cell. Let $T$ be a $C^{3}$ triangulation of $N^{3}$ of mesh less than $\varepsilon$; by $[11$, p. $78,(3.4)]$ we may suppose that $f$ is transverse regular on each simplex of each dimension. As in the proof of 3.3 for each $r$-simplex $\sigma^{r}(r=0,1,2), f^{-1}\left(\sigma^{r}\right) \approx \sigma^{r}$, and $f^{-1}\left(\partial \sigma^{3}\right) \approx \partial \sigma^{3}$. From the transverse regularity $f^{-1}\left(\partial \sigma^{3}\right)$ is bicollared, so that [4] $f^{-1}\left(\sigma^{3}\right) \approx \sigma^{3}$. Thus the $f^{-1}\left(\sigma^{r}\right)$ define a $C^{3}$ triangulation of $M^{3}$, and a simplicial homeomorphism $g: M^{3} \rightarrow N^{3} ; h$ results from [21].

In general $\operatorname{dim} n$, this argument, using induction, fails, since it is not clear that

$$
f \mid f^{-1}\left(\text { int } \sigma^{r}\right): f^{-1}\left(\text { int } \sigma^{r}\right) \rightarrow \text { int } \sigma^{r}
$$

is cellular for $r<n$. If the Poincaré Conjecture is true in dimensions 3 and 4 , it is.

4. A differential characterization of monotone maps. The hypothesis $J \not \equiv 0$ is needed [5, p. 707, (11)].

4.1. ReMARK. Let $M^{n}$ and $N^{n}$ be oriented manifolds, and let $f$ be $C^{1}$, not necessarily onto, such that the Jacobian determinant $J \not \equiv 0$ on every nonempty open set. Then $f$ is monotone onto if and only if $\operatorname{deg} f=1$ and $J \geqq 0$ or $\operatorname{deg} f=-1$ and $J \leqq 0$.

Proof. "Only if" is immediate from Sard's Theorem [28, p. 47, Theorem 3.1] and [28, p. 127, Theorem 4.2].

Suppose $\operatorname{deg} f=1, J \geqq 0$, and $f$ is not monotone. Then for some $\bar{y} \in N^{n}, f^{-1}(\bar{y})$ has at least two components $Y_{1}$ and $Y_{2}$. Choose a closed $n$-ball $U$ such that $Y_{1}$ and $Y_{2}$ are in different components $U_{1}$ and $U_{2}$ of $f^{-1}(U)$. Then $f\left(\right.$ bdy $\left.U_{i}\right) \subset$ bdy $U$, and the local degree $\mu\left(y, f, U_{i}\right)[23, \S I I .2]$ is independent of $y \in$ int $U$. Let $w \in$ int $U$ be a regular value $\left[28\right.$, p. 47]; from the hypotheses, $w \in f\left(U_{i}\right)$ for at most one $i$-say $w \notin f\left(U_{1}\right)$. Thus $\mu\left(w, f, U_{1}\right)=0$, so that $\mu\left(y, f, U_{1}\right)=0$ for every $y \in$ int $U$. Since $J \mid U_{1} \not \equiv 0$, there is a regular point $x \in$ int $U_{1}-f^{-1}\left(f\left(R_{n-1}\right)\right)$, so that $\mu\left(f(x), f, U_{1}\right)=1$, and a contradiction results. 
5. Improved hypotheses in [7].

5.1. REMARK. The differentiability hypotheses of various results in [7] can be improved, viz. $C^{n}$ can be replaced by

(1.3) $C^{n-q}([25]$ or [9]),

(1.5), (1.6), (1.7), (1.8), (1.9) $C^{2}$ (use above (1.3)),

(2.1) (the structure theorem for differentiable open maps) $C^{3}$ (proof below),

(2.2), (2.3) $C^{3}$ (use above (2.1)),

(2.5) $C^{3}$ (proof below).

In addition the hypothesis " $M^{n}$ is compact" in (1.8) and (2.1) can be replaced by " $f$ is proper".

Proof. (2.1) For the first conclusion, use the original proof, but with the above (1.3). For the second conclusion, follow the original proof through the last full paragraph on p. 93. It suffices to prove that $B_{f} \notin R_{n-3}(f)$; suppose the contrary.

Given $\bar{x} \in B_{f}$, choose a connected open neighborhood $V$ of $x$ such that $f(V)$ is an open $n$-ball and $g=f \mid V: V \rightarrow f(V)$ is a proper map [11, p. 74, (1.14)]. By above (1.3) $\operatorname{dim} R_{n-3}(f) \leqq \operatorname{dim} f\left(R_{n-3}(f)\right) \leqq n-3$, and since $f\left(R_{n-3}(f)\right)$ is closed $V-f\left(R_{n-3}(f)\right)$ is connected. By [9, Theorem 1] $V-f\left(R_{n-3}(f)\right)$ is simply connected, so that the covering map [22, p. 128] $g \mid\left(V-f^{-1}\left(f\left(R_{n-3}(f)\right)\right)\right)$ is a homeomorphism. Thus $g$ is a homeomorphism, contradicting the choice of $\bar{x}$.

(2.5) The argument is unchanged, except for the case $R_{n-1}(f) \subset R_{0}(f)$. Thus $B_{f} \subset R_{n-3}(f)$; by above (1.3) $\operatorname{dim} R_{n-3}(f) \leqq n-3$, by above (1.7) $f$ is open, and by the above (2.1) $f$ is a local homeomorphism, yielding the desired contradiction.

\section{REFERENCES}

1. S. Armentrout, Cellular decompositions of 3-manifolds that yield 3-manifolds, Bull. Amer. Math. Soc. 75 (1969), 453-456. MR 39 \#935.

2. -_- Upper semi-continuous decompositions of $E^{3}$ with at most countably many nondegenerate elements, Ann. of Math. (2) 78 (1963), 605-618. MR 27 \#6255.

3. A. Borel and A. Haefliger, La classe d'homologie fondamentale d'un espace analytique, Bull. Soc. Math. France 89 (1961), 461-513. MR 26 \#6990.

4. M. Brown, A proof of the generalized Schoenflies theorem, Bull. Amer. Math. Soc. 66 (1960), 74-76. MR 22 \#8470b.

5. P. T. Church, Differentiable maps with non-negative Jacobian, J. Math. Mech. 16 (1966), 703-708. MR 34 \#5096.

6. - Differentiable monotone maps on manifolds, Trans. Amer. Math. Soc. 128 (1967), 185-205. MR 39 \#3505.

7. - Differentiable open maps on manifolds, Trans. Amer. Math. Soc. 109 (1963), 87-100. MR 27 \#4245.

8. - Factorization of differentiable maps with branch set dimension at most $n-3$, Trans. Amer. Math. Soc. 115 (1965), 370-387. MR 33 \#3306.

9. — On points of Jacobian rank k. II, Proc. Amer. Math. Soc. 16 (1965), 1035-1038. MR 31 \#6241.

10. P. T. Church and W. D. Nathan, Real analytic maps on manifolds, J. Math. Mech. 19 (1969), 19-36. MR 39 \#4860.

11. P. T. Church and J. G. Timourian, Fiber bundles with singularities, J. Math. Mech. 18 (1968/69), 71-90. MR 38 \#3876. 
12. H. Kneser, Geschlossene Flächen in dreidimensionalen Mannigfaltigkeiten, Jber. Deutsch. Math.-Verein. 30 (1929), 248-260.

13. K. W. Kwun and F. Raymond, Almost acyclic maps of manifolds, Amer. J. Math. 86 (1964), 638-649. MR 32 \#1712.

14. S. MacLane, Homology, Die Grundlehren der math. Wissenschaften, Band 114, Academic Press, New York; Springer-Verlag, Berlin, 1963. MR 28 \#122.

15. D. R. McMillan, Jr., Acyclicity in three-manifolds, Bull. Amer. Math. Soc. 76 (1970), 942-964.

16. — Compact, acyclic subsets of three-manifolds, Michigan Math. J. 16 (1969), 129136. MR 39 \#4822.

17. — Decompositions of euclidean space yielding a manifold, Notices Amer. Math. Soc. 17 (1970), 679. Abstract \#70T-G81.

18. - Strong homotopy equivalence of 3-manifolds, Bull. Amer. Math. Soc. 73 (1967), 718-722. MR 37 \#4817.

19. J. Milnor, A unique decomposition theorem for 3-manifolds, Amer. J. Math. 84 (1962), 1-7. MR 25 \#5518.

20. J. R. Munkres, Elementary differential topology, Ann. of Math. Studies, no. 54, Princeton Univ. Press, Princeton, N. J., 1963. MR 29 \#623.

21. - Obstructions to the smoothing of piecewise-differentiable homeomorphisms, Ann. of Math. (2) 72 (1960), 521-554. MR 22 \#12534.

22. R. S. Palais, Natural operations on differential forms, Trans. Amer. Math. Soc. 92 (1959), 125-141. MR 22 \#7140.

23. T. Radó and P. V. Reichelderfer, Continuous transformations in analysis. With an introduction to algebraic topology, Die Grundlehren der math. Wissenschaften, Band 65, SpringerVerlag, Berlin, 1955. MR 18, 115.

24. J. H. Roberts and N. E. Steenrod, Monotone transformations of two-dimensional manifolds, Ann. of Math. (2) 39 (1938), 851-862.

25. A. Sard, Hausdorff measure of critical images on Banach manifolds, Amer. J. Math. 87 (1965), 158-174. MR 30 \#3958.

26. L. C. Siebenmann, Approximating cellular maps by homeomorphisms, Notices Amer. Math. Soc. 17 (1970), 532. Abstract \#674-40.

27. E. H. Spanier, Algebraic topology, McGraw-Hill, New York, 1966. MR 35 \#1007.

28. S. Sternberg, Lectures on differential geometry, Prentice-Hall, Englewood Cliffs, N. J., 1964. MR 33 \#1797.

29. R. Thom, Quelques propriétés globales des variétés différentiables, Comment. Math. Helv. 28 (1954), 17-86. MR 15, 890.

30. J. R. Walker, Monotone mappings and monotone decompositions, Ph.D. Dissertation, Syracuse University, Syracuse, New York, 1970.

31. H. Whitney, Differentiable manifolds, Ann. of Math. (2) 37 (1936), 645-680.

32. G. T. Whyburn, Analytic topology, Amer. Math. Soc. Colloq. Publ., vol. 28, Amer. Math. Soc., Providence, R. I:, 1942. MR 4, 86.

33. A. Wright, Mappings from 3-manifolds onto 3-manifolds (to appear).

34. - Monotone mappings of compact 3-manifolds, Notices Amer. Math. Soc. 16 (1969), 853-854. Abstract \#69T-G121.

SYRACUSE UNIVERSITY, Syracuse, NeW York 13210 